\title{
Tailoring Optical Bistability In Nonlinear Photonic Crystals Based on Metallic Nanoparticles And Graphene
}

Tayebeh Naseri ( $\sim$ tayebe.naseri@gmail.com )

Razi University https://orcid.org/0000-0003-2941-9051

Hamid Pashaei Adl

Razi University

\section{Research Article}

Keywords: Nonlinear Photonic Crystals (NPC), Optical Bistability (OB), Graphene, Metallic Nanoparticles (MNs).

Posted Date: December 10th, 2021

DOI: https://doi.org/10.21203/rs.3.rs-736463/v1

License: (c) (i) This work is licensed under a Creative Commons Attribution 4.0 International License.

Read Full License 


\title{
Tailoring Optical Bistability in Nonlinear Photonic Crystals Based on Metallic Nanoparticles and Graphene
}

\author{
Tayebeh Naseri* and Hamid Pashaei Adl \\ Department of Physics, Razi University, Kermanshah, Iran and \\ Instituto de Ciencia de Materiales (ICMUV), Universidad de Valencia, \\ C/ Catedrtico Jos Beltrn, 2, E-46980 Paterna, Spain.
}

\section{ABSTRACT}

In this work, we revisit the optical response of a one-dimensional photonic crystal consisting of graphene monolayers and a plasmonic nanocomposite as a defect layer in the structure. By taking advantage of the modified transfer matrix approach, the analytical solution of the light transmission and field distribution of the photonic crystal are evaluated. Besides, by considering one of the layers as a Kerr-nonlinear medium, we delve into optical bistability phenomenon in the model for two different cases. Our numerical results reveal that the proposed photonic crystal can enhance the field distribution and reduce the optical bistability's threshold in comparison to the conventional photonic crystals. Furthermore, the optical bistable switch-up and switch-down thresholds of the proposed resonator can be tailored flexibly by plasmon-plasmon interactions in the defect layer. Finally, the electric field distribution amelioration and optical bistability by means of graphene layers in the structure are attainable. The influences of the parameters such as the graphene and the nanocomposite on the performance of $\mathrm{OB}$ are analyzed and compared in the two different cases. Therefore, present approach can lay the groundwork for designing highly sensitive surface plasmon resonance biosensors and switches where the proposed technique may find unprecedented capabilities.

Keywords: Nonlinear Photonic Crystals (NPC); Optical Bistability (OB); Graphene; Metallic Nanoparticles (MNs).

\section{INTRODUCTION}

Photonic crystals (PCs) have been considered as one of the most appealing structures due to innumerable different materials which can be used in designing the photonic crystals such as linear and nonlinear dielectric materials [4], metals [5], graded-index materials [6] and metamaterials [7]. Based on structural arrangement, materials used in the structure and the number of underlying structural layers, photonic crystals demonstrate

\footnotetext{
*Electronic address: tayebe.naseri@gmail.com
}

mesmerizing optical responses. The nonlinear optical responses of the photonic crystal has given the momentum for exploring its applications in designing all-optical devices in optics communication and information arenas.

Nonlinear photonic crystals (NPCs) pave the way to improve and modify nonlinear optical processes. Various nonlinear optical phenomena such as nonlinear frequency conversion [8], second and high harmonic generation[9], four-wave mixing [10] and optical bistability $[11,16,17]$ have been explored in photonic crystals both theoretically and experimentally.

Optical bistability (OB) as the existence of two stable output states of an input light intensity is an important means to control light propagation with another light. This has been studied in various media such as multi-level atomic systems[18], Graphene and plasmonic nanostructures [19], and NPCs [11, 12]. The optical bistability and multistability in a defect structure doped with polaritonic materials and three-level nanoparticles have been investigated recently [13-15]. It was shown that the threshold of optical bistability can be manipulated by some controllable parameters such as Rabi frequency, line width of upper level, and thickness of defect structure. The OB can be realized by the dynamic shifting of band edges in NPCs. In OB phenomenon, the system shows two stable outputs for a specific input in presence of optical nonlinearity and positive feedback of the structure. Needless to mention that inquiring new optical materials with tunable parameters is the cornerstone for dynamically tunable optical bistable devices.

Graphene as a 2D material consisting of one layer of carbon atoms arrayed in a hexagonal lattice has a broadband and tunable optical response in the IR to visible frequency range [20-22]. It further reveals extremely large Kerr nonlinearity due to the linear band structure near the Fermi energy level that has been explored widely [23-25]. Fascinatingly, the bistablity behavior in the graphene-based structures can be electrically controlled and tuned just by changing the applied voltage on the graphene.

On the other hand, recent advanced progresses in nanotechnology have shown that metallic nanoparticles have emerged as powerful structures in various optical applications as a result of their superb optical properties[26-28]. That is to say, the optical properties 
of metallic nanoparticles are explained through surface plasmon resonances (SPRs) strongly depending on various parameters such as nanoparticles size, shape, concentration and spatial distribution as well as the properties of the background medium [29, 30].

The remarkable optical properties of metallic nanoparticles have been studied extensively and metal-dielectric composites have found a plethora of applications in nanophotonic [31-33]. The unique optical characteristics of these composite structures can be achievable as the contribution of the local field of the nanoparticles is tunable which is one of prerequisites of designing nonlinear optical devices.

Combining the nonlinearity of graphene and plasmonic excitation properties of metallic nanocomposites together have come forth to study Kerr nonlinearity and consequently OB phenomena. In this work, we study a graphene monolayer in a $1 \mathrm{DPC}$ and investigate the formative impact of graphene on the transmission properties. We stumble on that graphene significantly modifies the characteristics of the optical bistability behavior. A shift in defect modes and consequently $\mathrm{OB}$ can be seen via changing the refractive index and the light localization in the nonlinear defect layer.

Creating such a strong light localization prepares the ground for the tunable and low threshold OB with wide bistability hysteresis loop in the strong light matter interaction regime in the PCs. metallic nanocomposite and graphene are a big boon for strengthening the interaction between light and the PC.

In this manuscript, we present a new scheme to enhance the optical response of a one-dimensional photonic crystal that consists of graphene monolayers and a plasmonic nanocomposite as a defect layer in the structure. The numerical results demonstrate that the proposed photonic crystal can enhance the field distribution and reduce the optical bistability threshold in comparison with the common photonic crystals. The nonlinear photonic crystal coupled with graphene sheets and metallic nanocomposite sets the stages for the bistable response of the transmitted light intensity. Realizing a low intensity threshold of $\mathrm{OB}$ is a critical challenge in scheming nonlinear devices and decreasing the switching threshold becomes practical with this NPC structure; with this end in view, such this controllable bistable system could find potential applications in optical all-optical switching and sensing.

\section{THEORETICAL MODEL AND METHODS}

In this study, we consider two different structures, as shown in Fig.1, for Case'I' the proposed structure is $(B A)^{N} D(A B)^{N}$, where $A, B$ are isotropic dielectric layers with high and low refractive indexes, respectively and
$N$ is the number of periods. The interfaces of the layers are parallel to the $(x-y)$ plane, and the $z$ axis is normal to the structure. In Case'II', it is assumed that the linear

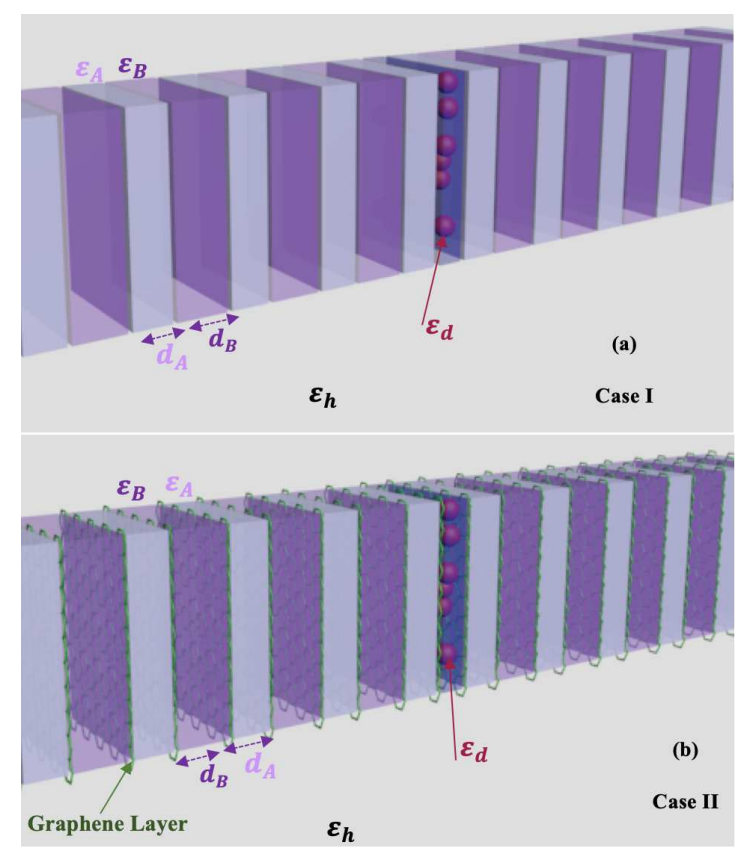

FIG. 1: Schematic structures for two different cases of $1 \mathrm{D}$ defective photonic crystal.

graphene monolayers $G$ are embedded between adjacent dielectric layers (see Fig. 2). The defect layer $D$ is a nanocomposite with a refractive index distribution function $\epsilon_{D}$ based on Maxwell Garnett approximation[34]:

$$
\epsilon_{D}=\frac{\epsilon_{d} \epsilon_{h}(1+2 f)+2 \epsilon_{h}^{2}(1-f)}{\epsilon_{d}(1-f)-\epsilon_{h}(2+f)}
$$

where the dielectric function of a plasmonic noble metal is described by the Drude model and given by $\epsilon_{d}=$ $\epsilon_{\infty}-\frac{\omega_{p}^{2}}{\left(\omega^{2}+i \gamma \omega\right)}$, and $\omega_{p}, \gamma$ and $\epsilon_{\infty}$ are the plasma frequency of the metal, the collision frequency of free electrons and the high-frequency part of the dielectric function[35]. In this work, gold $(\mathrm{Au})$ is used due to its strong plasmon resonance with tunable resonance wavelength [36]. Also, $\epsilon_{h}, f$ are permittivity of host material and metal filling fraction of defect layer, respectively. In this paper, the graphene monolayer is described by its relative permittivity $\epsilon_{G}=1+\frac{i \sigma_{G} \eta_{0}}{k_{0} d_{G}}$. The frequency dependent surface conductivity of graphene $\sigma_{G}$ is written as the superposition of the intraband $\sigma_{\text {intra }}$ and the interband term $\sigma_{\text {inter }}[19]$, which is expressed in the following forms:

$$
\sigma_{G}=\sigma_{\text {intra }}+\sigma_{\text {inter }}
$$




$$
\begin{array}{r}
\sigma_{\text {intra }}=\frac{i e^{2} k_{B} T}{\pi \hbar^{2}(\omega+i / \tau)}\left[\frac{E_{F}}{k_{B} T}+2 \operatorname{Ln}\left(e^{\frac{-E_{F}}{k_{B} T}}+1\right)\right], \\
\sigma_{\text {inter }}=\frac{i e^{2}}{4 \pi \hbar} \operatorname{Ln}\left[\frac{2 E_{F}-(\omega+i / \tau)}{2 E_{F}+(\omega+i / \tau)}\right]
\end{array}
$$

where $\omega, E_{F}, \tau, T, e, k_{B}, \eta_{0}, d_{G}, \hbar$, and $k_{0}=2 \pi / \lambda$ are the frequency of the incident field, the Fermi energy, the electron-phonon relaxation time, the temperature, the elemental electron charge, the Boltzmann constant, the impedance of air, the thickness of the graphene nanolayer, the reduced Planck constant, and the vacuum wavevector, respectively. It is worth mentioning that only the transverse electric field $(T E)$ is being studied here. So, the electric fields in layers are written as:

$$
\vec{E}_{j}(x, z)=E(z) e^{i\left(k_{x} x+k_{z j} z\right)} \hat{e}_{y}
$$

Here, $k_{x}=k_{0} \sin (\theta)$, with $k_{0}=\omega / c$, and $k_{z j}=$ $k_{0} \sqrt{\epsilon_{j} \mu_{j}\left(1-\sin ^{2}(\theta) / \epsilon_{j} \mu_{j}\right)} . \quad \theta$ is the angle of the incident light. Inside the layers, the electric field is governed by the Helmholtz equation [37]:

$$
\frac{d^{2} E}{d z^{2}}+\left(\epsilon_{j} \mu_{j} \omega^{2} / c^{2}-k_{x}^{2}\right) E=0
$$

The subscript $j$ denotes the number of the layers. At the interface between two layers, the boundary conditions are imposed [38-40]:

$$
\mathbf{m}_{\mathbf{l}}\left(\boldsymbol{\Delta}_{\mathbf{j}}\right)=\left(\begin{array}{cc}
\cos \left(k_{z j} \Delta z_{j}\right) & -\frac{\mu_{j} \omega}{k_{z j} c} \sin \left(k_{z j} \Delta z_{j}\right) \\
\frac{k_{z j} c}{\mu_{j} \omega} \sin \left(k_{z j} \Delta z_{j}\right) & \cos \left(k_{z j} \Delta z_{j}\right)
\end{array}\right)
$$

Here, $l=1,2, \ldots, N$, where $N$ is the total number of the layers in the structure. Consequently, we have the total transfer matrix:

$$
M^{\text {tot. }}=\prod_{l=1}^{N} m_{l}\left(\Delta z_{j}\right)
$$

The tangential components of the electric and magnetic fields at the incident site $z=0$ and the transmitted site $z=L$ are related by the following matrix equation:

$$
\left(\begin{array}{c}
E_{y_{1}} \\
H_{x_{1}}
\end{array}\right)_{z=0}=M^{\text {tot. }}\left(\begin{array}{c}
E_{y_{N}} \\
H_{x_{N}}
\end{array}\right)_{z=L}
$$

Finally, the transmission can be obtained as $T=t t^{*}$ where $t=2 p /\left(p M_{11}^{\text {tot. }}+p M_{22}^{\text {tot. }}+p^{2} M_{12}^{\text {tot. }}+M_{21}^{\text {tot. }}\right)$ and $p=\cos (\theta)$. It is important to note that subscript " $\mathrm{j} "$ in equations 7,8 represents the linear layer $(\mathrm{A}, \mathrm{G}, \mathrm{D})$ with thicknesses of dA, dD, and dG. However, in the nonlinear layer (B), the subscript " $j$ " refers to very thin sublayers with a thickness of $\mathrm{dB} / \mathrm{N}_{\text {sub }}$., where $\mathrm{N}_{\text {sub }}$. denotes the number of sublayers. It is possible to use the same transfer matrix for both linear and nonlinear regimes by using this strategy (dividing the nonlinear layer into very thin sublayers) [6, 41, 42].

\section{RESULTS AND DISCUSSION}

As mentioned earlier, in both cases (Case'I'and Case'II') the thickness of the layers are fixed to $d_{A}=80 \mathrm{~nm}, d_{B}=120 \mathrm{~nm}, d_{D}=20 \mathrm{~nm}, d_{G}=0.33 \mathrm{~nm}$ and $f=0.1$. It is assumed that the layers are nonmagnetic and their permeabilities are $\mu_{A}=\mu_{B}=\mu_{D}=1$.

\section{A. Case ' $I$ '}

For the first case, we study nonlinear properties of the structure without graphene sheets, and the optical parameters of the system are chosen as: $N=5$, $\epsilon_{A}=6.25, \epsilon_{h}=2.89$ and $\epsilon_{B}=2.25+\chi^{(3)}|E|^{2}$. Although negative Kerr nonlinearity has been experimentally proven in various composite crystals [43-46], the majority of naturally occurring materials have a positive Kerr coefficient, so here $\chi^{(3)}$ is considered as a small positive, third-order susceptibility coefficient accounting for the nonlinear interactions in the structure and $E$ is the electric field. The optical Kerr effect is a change in the refractive index of a medium caused directly by the electric field of incident light. The importance of this type of nonlinearity in PCs has been demonstrated in the literature when designing several nonlinear devices based on the optical Kerr effect, such as optical diodes [47-49], switches, and limiters [50, 51].

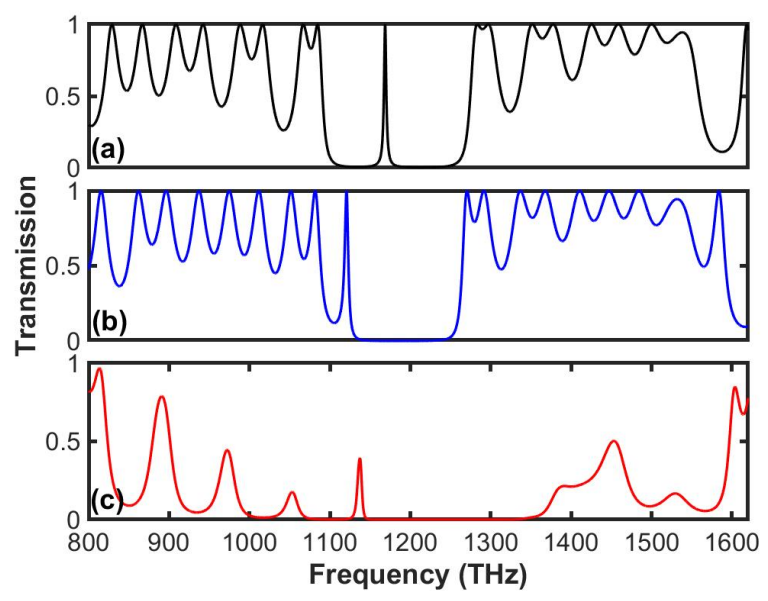

FIG. 2: The transmission spectrum of the defective structure $(B A)^{5} D(A B)^{5}$ at normal incidence for (a) a defect free structure $\mathrm{d}_{D}=0(\mathrm{~b})$ a dielectric defect layer with $\epsilon_{D}=\epsilon_{h}(\mathrm{c})$ a nanocomposite defect layer.

Fig.2 shows the effect of the nanocomposite defect layer on the band gap of the structure. In Fig. 2(a) the transmission spectrum of a defect free structure plotted to draw an analogy with defective structures, it is obvious from this figure that by considering a defect layer in the middle of the structure the lower edge of the band gaps a bit shifted to the lower energies and by changing the 
electric permittivity of defect layer from host material to the permittivity of nanocomposite the band gap is broadened. The optical responses of the metallic nanocomposite comes from the surface plasmons that are collective oscillations of the conduction electrons in metallic particles, and by embedding the nanocomposite defect in the middle of the structure the light interaction with surface plasmons of metallic nano particles and also plasmonplasmon interactions would enhanced.Therefore, an enormous shift happens in the energy band gap and the energy band gap becomes wider (see Fig. 2(c)). To clarify the connection between the nanocomposite defect layer and the OB threshold intensity, the normalized transmitted light intensity $\left(\chi^{(3)}\left|E_{t}\right|^{2}\right)$ through the structure versus the normalized incident light intensity $\left(\chi^{(3)}\left|E_{i}\right|^{2}\right)$ are illustrated in Fig.3. Here, we consider the frequencies lying close to the low-frequency edges of the defect modes. By introducing a positive Kerr coefficient, the enhancement of the electric field (self-focusing effect) [52] occurs near the low-frequency edge of the defect mode which leads to the optical bistability. In the case of a negative Kerr coefficient, optical bistability can be seen only near the high-frequency edge where the electric field does not get enhanced (self-defocusing effect) [53].

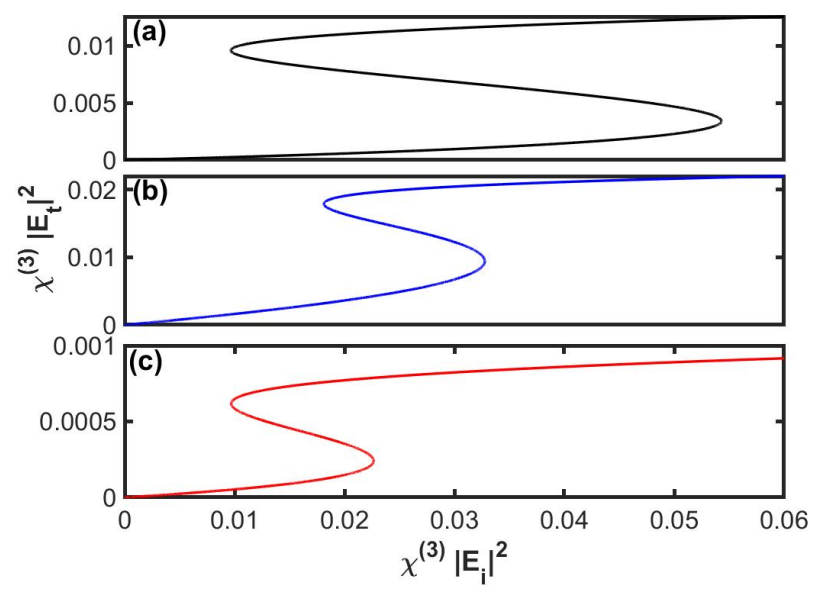

FIG. 3: Normalized transmitted light intensity $\left(\chi^{(3)}\left|E_{t}\right|^{2}\right)$ through the structure versus the normalized incident light intensity $\left(\chi^{(3)}\left|E_{i}\right|^{2}\right)(\mathrm{a}),(\mathrm{b})$, and (c) are corresponded to Fig.2.

According to this figure, there is a decrease in threshold intensity of OB by introducing the defect layer and this reduction in threshold intensity of OB intensify by embedding the nanocomposite layer, due to plasmonplasmon interactions in the defect layer. Such strong plasmon-plasmon interactions in the system allow an enhancement in optical nonlinearities due to the energy transfer between the plasmons. Moreover, the metallic nanoparticles are able to provide large tunability of the plasmon resonance wavelength which leads to boost the nonlinearity in the proposed structure, and as it is known, the bistable region and bistability threshold is strongly dependent on the nonlinearity of the structure. The ad- vantage of the defective structures over the defect-free structures is clearly demonstrated in Fig. 3 by comparing their OB threshold, so we will now focus on the defective structures in this paper.

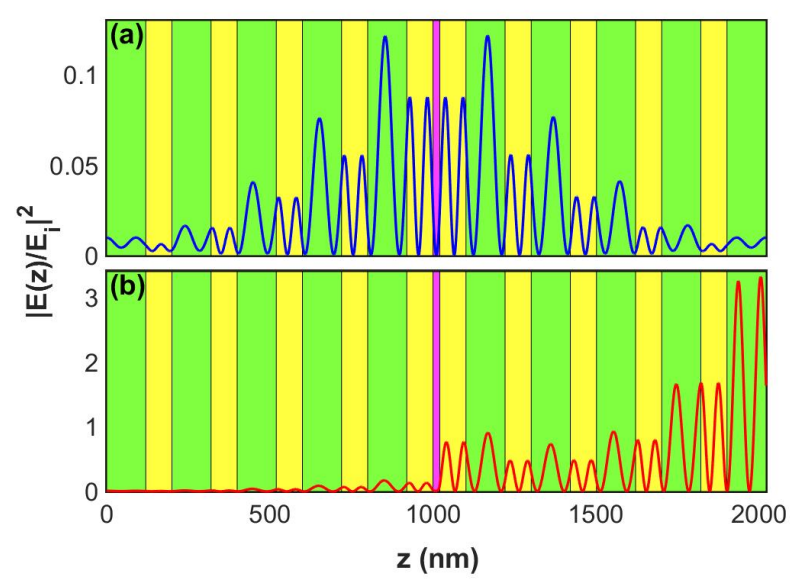

FIG. 4: Spatial distribution of the normalized transverse electric field intensity of a structure with (a) a dielectric defect layer with $\epsilon_{D}=\epsilon_{h}$ (b) a nanocomposite defect layer.

In Fig.4, we considered the normalized transverse electric field intensities of the proposed defective structure in the frequency of defect mode in two different conditions. As it is seen from the Fig.4a, for the ordinary defect layer the electric field intensity reaches the peak in the middle of the nonlinear layers and the magnitude of these peaks is maximized symmetrically in the vicinity of the defect layer.

On the contrary, when the structure includes nanocomposite defect layer, the electric field intensity inside the structure at the frequency of the defect mode for the situations described in Fig.2c, the field intensity grows gradually from the incident side to the transmitted side and this growth intensified after passing through the defect layer (Fig.4b).

To study the dependence of the photonic band gaps (PBGs) on the incidence angle $\theta$ and nano particles filling factor $f$, we plot the photonic band structure of the system as a function of $\theta$ and $f$ in figures 5 and 6 , respectively. Here, the gaps are represented by the dark areas while the bright areas show the allowed bands for the TE polarization. It is clear that by increasing the incident angle PBGs and the defect modes shift to the higher frequencies, moreover when defect layer contains metallic nanoparticles in large angles the defect mode disappears.

Figure 6 depicts how the filling factor of metallic nanoparticles affects defect mode and photonic bandgap, the defect mode sharpens and slightly moves to the lower energies when the filling factor is increased from 0.1 to 0.4 , meaning that the Q-factor of the defect modes, which is one of the main parameters in designing Bio-sensors and refractometers, can be improved by fine-tuning with the filling factor. Furthermore, changing the filling factor 


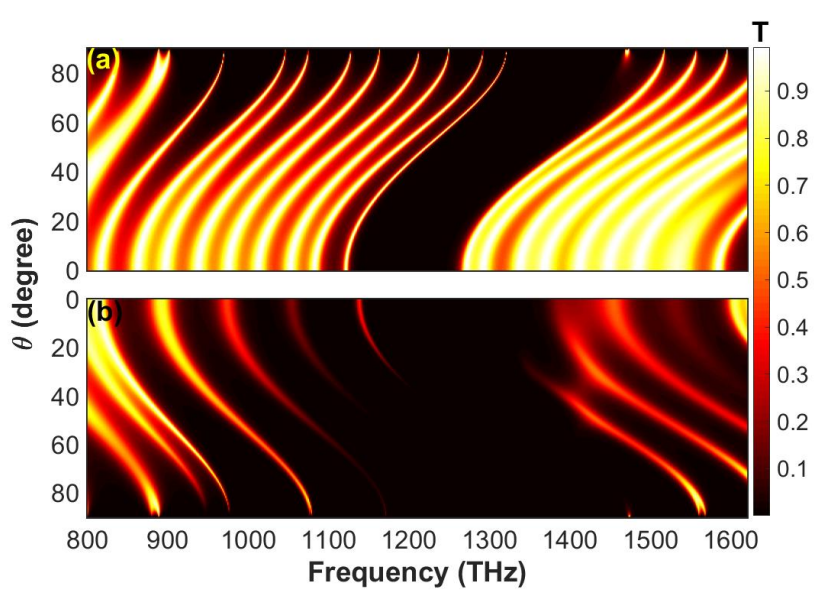

FIG. 5: photonic band gap of the defective structure with (a) a dielectric defect layer with $\epsilon_{D}=\epsilon_{h}$ (b) a nanocomposite defect layer.

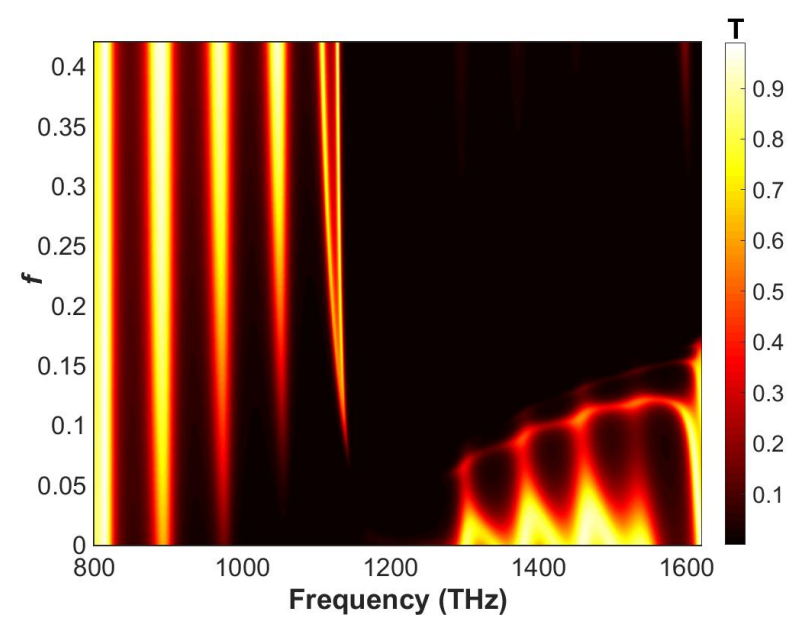

FIG. 6: The transmission spectra of the structure containing nanocomposite defect layer in the plane of frequency and filling factor.

severely affects the higher edge of the photonic bandgap; however, the lower edge does not exhibit high sensitivity to filling factor variance, making it a strong choice for designing low pass filters.

\section{B. Case 'II'}

In the second case, we studied the structure of Case 'I' with embedded linear graphene sheets between the layers (see Fig.1(b)).

The linear transmission of the graphene based structure has been represented in Fig. 7 as a function of frequency in comparison to the first case to investigate the effect of the graphene layers on the frequency of the defect mode when the structure includes nanocomposite. It is obvious that by embedding graphene sheets between the layers, the defect mode slightly moves to the lower energies, since.

As shown in Eq. 2, the surface conductivity of graphene includes the imaginary part and the real part, which shows that graphene acts like a very thin metallic layer. The addition of graphene layers to the photonic crystal leads to extra positive phase shift and energy loss because of the real part and the imaginary part of the surface conductivity of graphene.

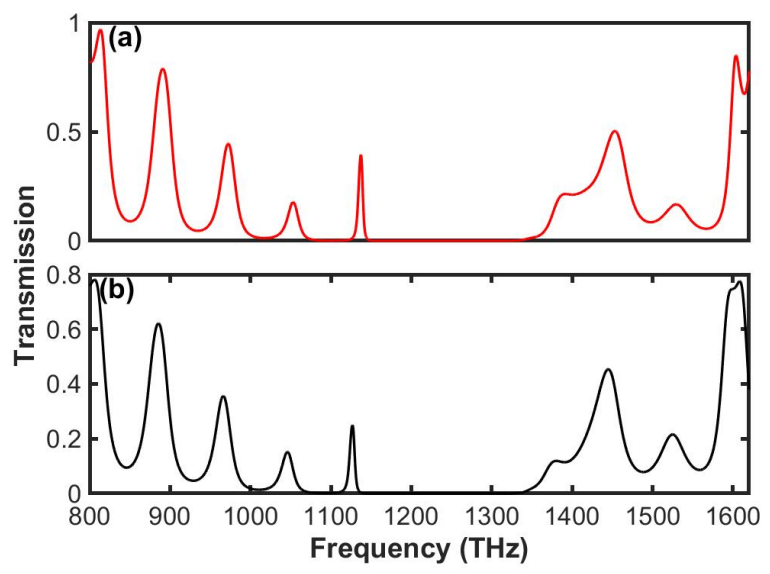

FIG. 7: The transmission spectrum of the defective structure at normal incidence for (a) $(B A)^{5} D(A B)^{5}$ (b) $(G B G A)^{5} G D G(A G B G)^{5}$ with other parameters unchanged. In both cases defect layer is nanocomposite

Figure. 8 shows the effect of linear graphene monolayers on nonlinear response of the system when the structure consists of a nanocomposite defect layer. In this figure the normalized transmitted light intensity $\left(\chi^{(3)}\left|E_{t}\right|^{2}\right)$ through the structure has been plotted versus the normalized incident light intensity $\left(\chi^{(3)}\left|E_{i}\right|^{2}\right)$.

In line with this figure, threshold intensity of OB declined dramatically by inserting graphene sheets between the layers. It should be noted that the OB hysteresis loop shrinks. In the presence of graphene monolayers in the photonic crystal, the nonlinearity of this structure would be enhanced considerably.

To investigate the effect of graphene sheets on the behavior of electric field distribution inside the defective $\mathrm{PC}$ we made an analogy between two structures related to the Fig. 7. Both of the structures defect layer consist of metallic nanoparticles and the geometry of the structures are the same except in one of them graphene monolayers are inserted between the layers. As shown in Fig. 9, in both structures, the field intensity is being enhanced from the incident side to the transmitted side and this growth intensified after passing through the nanocomposite layer, but enhancement of the electric field intensity is higher in the graphene-based PC. 


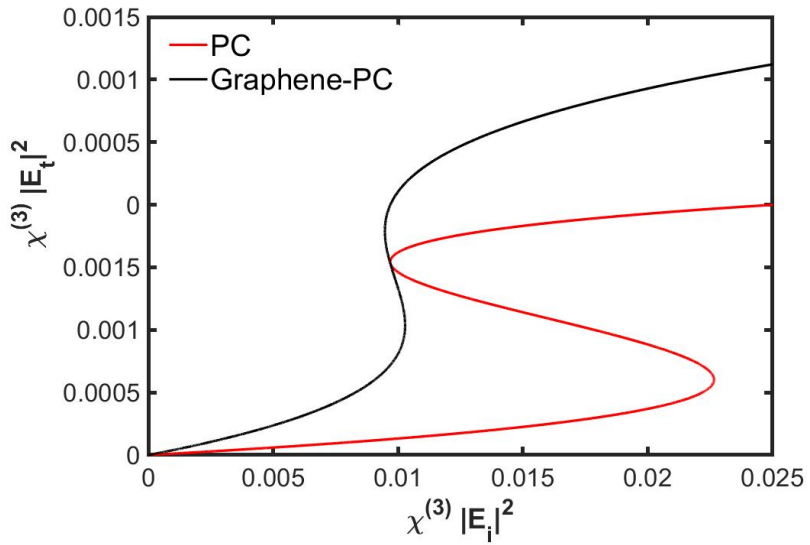

FIG. 8: Transmitted light intensity $\left(\chi^{(3)}\left|E_{t}\right|^{2}\right)$ through the structure versus the incident light intensity $\left(\chi^{(3)}\left|E_{i}\right|^{2}\right)$; keeping the other parameters unchanged

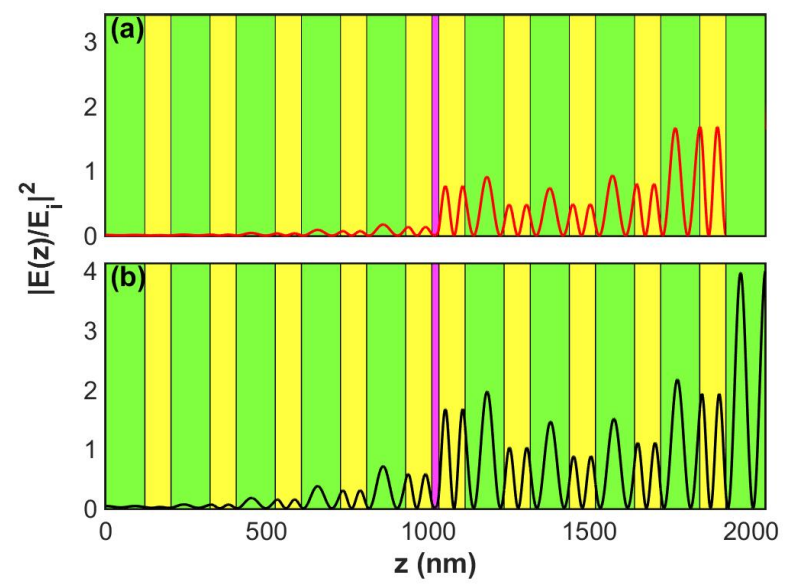

FIG. 9: Spatial distribution of the normalized transverse electric field intensity of structure corresponded to Fig.8. (a) without graphene, (b) with graphene; the other parameters unchanged

\section{CONCLUSION}

In conclusion, we have investigated the issue of optical transmission and optical bistability in the one dimensional photonic crystal. In this model, the defect mode is deemed as a metallic nanocomposite including spherical golden nanoparticles. We have shown that tailoring of the photonic crystal in both cases (with and without graphene monolayers) allows to obtain the high optical responses. The numerical results demonstrate that a tremendous shift happens in the energy band gap and consequently the energy band gap becomes squarely wider. That is to say, light intensity enhancement stems from surface plasmon resonances in graphene and plasmonic nanoparticles embedded in the defect layer. This exceptional phenomenon reduces the threshold input intensity in the OB hysteresis loop as well.

\section{REFERENCES}

[1] P. Hou, Y. Chen, X. Chen, J. Shi, and Q. Wang, Anomalous bistable shift for a one-dimensional photonic crystal doped with a sub- wavelength layer and a nonlinear layer, Phys Rev A 75, 045802 (2008).

[2] E. Ozbay, Science, 311, 189 (2006).

[3] K. V. Sreekanth, A. De Luca, and G. Strangi, Appl Phys Lett, 104, 171904 (2014).

[4] B. Mandal, A.R. Chowdhury, Spatial soliton scattering in a quasi phase matched quadratic media in presence of cubic nonlinearity, J Electromagn Waves Appl, 21(1), 123135 (2007).

[5] M. C. Larciprete, et al, J Appl Phys, 93, 5013 (2003).
[6] S. Roshan Entezar, Optical bistability in one-dimensional photonic band gap structure with nonlinear graded-index defect layer, Optics Communications, 287, 1924 (2013).

[7] J. Li, L. Zhou, C.T. Chan, and P. Sheng, "Photonic band gap from a stack of positive and negative index materials," Phys Rev Lett, 90, 083901 (2003).

[8] M-L. Ren, and Zh-Y. Li , Enhanced nonlinear frequency conversion in defective nonlinear photonic crystals with designed polarization distribution, J Opt Soc Am B, 27, 8 (2010).

[9] F. Asadollah Zarif, M. Khazaei Nezhad, and H. Rastegrar Moghaddam Rezaieun, Enhancement of efficiency of second-harmonic generation from MoS2 monolayers in 1D Fibonacci photonic crystals, Photonics and Nanos- 
tructures, (2019).

[10] J. Li, L. OFaolain, I. H. Rey, and Th. F. Krauss, Fourwave mixing in photonic crystal waveguides: slow light enhancement and limitations, Opt. Express 19, 44584463 (2011).

[11] K. V. Sreekanth, A. R. Rashed, A. Veltri, M. ElKabbash , and Giuseppe Strangi, Optical bistability in $\mathrm{Ag}-\mathrm{Al} 2 \mathrm{O} 3$ one-dimensional photonic crystals, EPL, 112, 14005 (2015).

[12] Y. Xu, B. Wan, Z. Zhou, Y. Ma, H. Zhang, and D. Zhang, "Tunable and asymmetric optical bistability of one-dimensional photonic crystals based on $\mathrm{InSb}$ and nonlinear materials," Applied Optics 59,31, (2020).

[13] G. Solookinejad, M. Panahi, E. Ahmadi Sangachin, and S. H. Asadpour, Observation of Optical Bistability in a Polaritonic Material Doped with Nanoparticles, Plasmonics 12, 18811887 (2017).

[14] The European Physical Journal Plus 131 (11), 1-6 (2016).

[15] S. H. Asadpour, and H. Rahimpour Soleimani; Transmission and reflection properties of incident pulse in a dielectric slab doped with quantum dot, Superlattices and Microstructures 62, 217-224 (2013).

[16] E. Centeno, and D. Felbacq, Phys Rev B, 62, 7683 (2000).

[17] M. Soljac, M. Ibanescu, S. G. Johnson, Y. Fink, and J. D. Joannopoulos, Optimal bistable switching in nonlinear photonic crystals, Phys Rev E, 66, 05560 (2002).

[18] T. Naseri, S. H. Asadpour, and R. Sadighi-Bonabi, "Some optical properties of four-level media via coherent and incoherent pumping fields," J. Opt. Soc. Am. B 30, 641-648 (2013).

[19] T. Naseri, and M. Balaei, "Enhanced nonlinear optical response of coreshell graphene-wrapped spherical nanoparticles," J. Opt. Soc. Am. B 35, 2278-2285 (2018).

[20] A. N. Grigorenko, M. Polini, and K. S. Novoselov; "Graphene plasmonics," Nature Photonics volume 6, 749758 (2012).

[21] A. K. Geim, and K. S. Novoselov; "The rise of graphene," Nanoscience and Technology,11-19 (2009).

[22] C. N. R. Rao, K. Biswas, K. S. Subrahmanyam, and A. Govindarajab "Graphene, the new nanocarbon," J. Mater. Chem., 19, 2457-2469 (2009).

[23] T. Christensen, W. Yan, A-P. Jauho, M. Wubs, and N. Asger Mortensen; "Kerr nonlinearity and plasmonic bistability in graphene nanoribbons," Phys. Rev. B 92, 121407(R) (2015).

[24] S.Thakur, B. Semnani, S. Safavi-Naeini \& A- H. Majedi; "Experimental Characterization of the Ultrafast, Tunable and Broadband Optical Kerr Nonlinearity in Graphene," Scientific Reports 9, 10540 (2019).

[25] D. B. S. Soh, R. Hamerly, and H. Mabuchi; "Comprehensive analysis of the optical Kerr coefficient of graphene," Phys. Rev. A 94, 023845 (2016).

[26] O-V Salata; "Applications of nanoparticles in biology and medicine," Journal of Nanobiotechnology, 2, 3 (2004).

[27] P. Ghosh, G. Han, M. De, Ch K.Kim, and V. M. Rotello; "Gold nanoparticles in delivery applications," 60, 11, 1307-1315 (2008).

[28] W. J. Stark, P. R. Stoessel, W. Wohlleben and A. Hafner; "Industrial applications of nanoparticles," Chem. Soc. Rev., 44, 5793-5805 (2015).

[29] E. A. Coronado, E. R. Encina and F. D. Stefani; "Optical properties of metallic nanoparticles: manipulating light, heat and forces at the nanoscale," Nanoscale,3, 4042-4059 (2011).

[30] Y. Leroux, J. Ch. Lacroix, C. Fave, G.Trippe, N. Flidj, J. Aubard, A. Hohenau, and J. R. Krenn; "Tunable Electrochemical Switch of the Optical Properties of Metallic Nanoparticles," ACS Nano 2, 4, 728732 (2008).

[31] "Applications of biosynthesized metallic nanoparticles A review,"

[32] M. A. Garcia; "Surface plasmons in metallic nanoparticles: fundamentals and applications," J. Phys. D: Appl. Phys. 44283001 (2011).

[33] T. Yonezawa; "Application 78 - Preparation of Metal Nanoparticles and Their Application for Materials," Nanoparticle Technology Handbook (Third Edition) 829837 (2018).

[34] M. Y. Koledintseva, R. E. DuBroff, and R. W. Schwartz; "a maxwell garnett model for dielectric mixtures containing conducting particles at optical frequencies," Progress In Electromagnetics Research, PIER 63, 223242, (2006).

[35] Y. Hashimoto, G. Seniutinas, A. Balytis, S. Juodkazis, and Y. Nishijima " $\mathrm{Au}-\mathrm{Ag}-\mathrm{Cu}$ nano-alloys: tailoring of permittivity," Scientific Reports 6, 25010 (2016).

[36] R. Sardar, A. M. Funston, P. Mulvaney, and R. W. Murray; "Gold Nanoparticles: Past, Present, and Future," Langmuir 25, 24, 1384013851(2009).

[37] S. M. Wang and L. Gao, "Nonlinear responses of the periodic structure composed of single negative materials", Optics Communication, 281, 197-204, (2006).

[38] M. Cuevas, "Surface plasmon enhancement of spontaneous emission in graphene waveguides", Journal of Optics, 18, 105003, (2016).

[39] P. Yeh, "Optical waves in layered media", Wiley Online Library, 95, (1988).

[40] H. Pashaei Adl, F. Bayat, N. Ghorani, S. AhmadiKandjani, and H. Tajalli, "A Defective 1-D Photonic Crystal-Based Chemical Sensor in Total Internal Reflection Geometry", IEEE Sensors, 17, 4046-4051, (2017).

[41] Entezar, S Roshan and Karimi, M Habil and Adl, H Pashaei, "Optical isolation via one-dimensional magnetophotonic crystals containing nonlinear defect layer", Optics Communications, 352, 91-95, (2015).

[42] Wang, SM and Gao, L, "Nonlinear responses of the periodic structure composed of single negative materials", Optics Communications, 267, 197-204, (2006).

[43] Samoc, M and Samoc, A and Luther-Davies, B and Woodruff, M, "The concept of guiding light with light and negative third-order optical nonlinearities of organics", Pure and Applied Optics: Journal of the European Optical Society Part A, 5, 681, (1996).

[44] Ohke, Shigeaki and Umeda, Tokuo and Cho, Yoshio, "Power-limiting action of optical waveguide having negative nonlinear claddings", Japanese journal of applied physics, 37, L1312, (1998).

[45] Maeda, Yoshinobu, "Optical bistability derived from the negative nonlinear absorption effect in erbium doped materials", Materials Science and Engineering: B, 81, 174$175,(2001)$.

[46] Helseth, LE, "Breaking the diffraction limit in nonlinear materials", Optics communications, 256, 435-438, (2005).

[47] Scalora, Michael and Dowling, Jonathan P and Bowden, Charles M and Bloemer, Mark J, "Optical limiting and switching of ultrashort pulses in nonlinear photonic band gap materials", Physical review letters, 73, 1368, (1994). 
[48] Fogel, Ishella S and Bendickson, Jon M and Tocci, Michael D and Bloemer, Mark J and Scalora, Michael and Bowden, Charles $\mathrm{M}$ and Dowling, Jonathan $\mathrm{P}$, "Spontaneous emission and nonlinear effects in photonic bandgap materials", Pure and Applied Optics: Journal of the European Optical Society Part A, 7, 393, (1998).

[49] Zhao, Nian-Shun and Zhou, Hui and Guo, Qi and Hu, Wei and Yang, Xiang-Bo and Lan, Sheng and Lin, XuSheng, "Design of highly efficient optical diodes based on the dynamics of nonlinear photonic crystal molecules", JOSA B, 23, 2434-2440, (2006).

[50] Bushuev, Vladimir Alekseevich and Pryamikov, Andrey Dmitrievich, "Effect of cubic nonlinearity on reflection and transmission of laser radiation in a one-dimensional photonic crystal", Quantum Electronics, 33, 515, (2003).

[51] Fedulova, Elena and Trubetskov, Michael and Amotchkina, Tatiana and Fritsch, Kilian and Baum, Peter and Pronin, Oleg and Pervak, Vladimir, "Kerr effect in multilayer dielectric coatings", Optics express, 24, 2180221817, (2016).

[52] Kominis, Y, "Analytical solitary wave solutions of the nonlinear Kronig-Penney model in photonic structures", Physical Review E, 73, 066619, (2006).

[53] Kominis, Y and Hizanidis, K, "Lattice solitons in selfdefocusing optical media: analytical solutions of the nonlinear Kronig-Penney model", Optics letters, 31, 28882890, (2006). 